\title{
IgG4-related disease: why is it so important?
}

\author{
MARTA LEGATOWICZ-KOPROWSKA
}

Department of Pathology, National Institute of Geriatrics, Rheumatology and Rehabilitation, Warsaw, Poland

\begin{abstract}
IgG4-related disease (IgG4-RD) is a recently defined systemic inflammatory and fibrous condition of unknown etiology and multiple clinical presentations. Characteristic features include elevated serum IgG4 levels in approximately 70\% of patients; diffuse lymphoplasmocytic infiltrates rich in IgG4(+) cells; a "storiform" fibrosis pattern; and obliterative phlebitis affecting various organs. The disease responds well to corticosteroid treatment, with a second-line therapy involving B-cell-directed immunosuppressive biologic agents. Despite intense studies, the pathogenesis of IgG4-RD remains unclear. The inflammatory infiltrates present in affected tissues contain also multiple polyclonal T and B cells, plasma cells, andoften - eosinophils. Cytokines secreted by type 2 helper T-cells and regulatory T-cells are known to cause $B$-cell differentiation into IgG4-producing plasma cells. On the other hand, large numbers of IgG4(+) plasma cells can be observed in nonspecific chronic inflammatory conditions, areas adjacent to neoplastic lesions with an inflammatory response, and in autoimmune inflammatory infiltrates. Thus, the fundamental question about the role of $I g G 4(+)$ cells in the pathogenesis of inflammation, tissue damage, and fibrosis in IgG4-RD still remains unanswered: does IgG4 stimulate or rather - which is more consistent with its natural properties - play a regulatory function in the inflammatory process?
\end{abstract}

Key words: diagnosis, pathogenesis, IgG4-related disease, IgG4 immunoglobulin.

(Centr Eur J Immunol 2018; 43 (2): 204-208)

\section{Introduction}

IgG4-related disease (IgG4-RD) is a newly classified, immunological condition. It involves potentially multiorgan inflammation and fibrosis, characterized (in most cases) by elevated serum IgG4 levels and presenting as tissue edema with fibrosis and marked inflammatory infiltration of IgG4-positive plasma cells. IgG4-RD constitutes a number of conditions previously classified separately according to the organ affected, therefore, it occurs in various forms: autoimmune pancreatitis type 1 (AIP1), Mikulicz's disease (MD), dacryoadenitis, sclerosing cholangitis, lymphadenopathy, and - less commonly - retroperitoneal fibrosis, Riedel's thyroiditis, sclerosing sialadenitis (Kuttner's tumor), and interstitial lung or kidney disease.

The key to diagnosis is histological examination showing inflammatory infiltrates of IgG4-positive cells, a characteristic pattern of fibrosis known as "storiform" fibrosis, a risk of venous involvement in the form of obliterative phlebitis, and possible eosinophilia. The presence of these characteristics in various organs, combined with elevated serum IgG4 levels was the basis for recognizing the observed organ lesions as manifestations of the same systemic disease.

Good therapeutic effects in IgG4-RD can be achieved with corticosteroids, unlike in the case of other conditions which need to be considered in the differential diagnosis, e.g. lymphoproliferative disease or Sjögren's syndrome.

\section{Pathogenesis}

The mechanisms of immune abnormalities underlying IgG4-RD remain unclear. The characteristic features of the condition are elevated serum IgG4 levels and profuse inflammatory infiltrates of IgG4-positive cells.

IgG4 antibodies are dynamic molecules - altering their properties by spontaneous exchange of one of the two Fab fragments between individual immunoglobulin molecules. This process involves dissociation of immunoglobulin G4 heavy-chain dimers and a subsequent bonding of each IgG4 half-molecule with a different IgG4 half-molecule. This half-molecule exchange yields bi-specific antibodies able to bind with two different antigens, but monovalent for each of them (Fig. 1). These properties of IgG4 molecules are the reason why IgG4 antibodies do not bind to the complement directly, do not initiate the classical pathway of complement activation, or why they are poor Fc receptor activators. This reduced IgG4 effector function has been responsible for these antibodies being considered anti-inflammatory [1]. IgG4 are believed to constitute a veritable antigen "garbage disposal" system, which can attenuate inflammation or protect against type I hypersensitivity by inhibiting IgE activity, as well as prevent type II and III hypersensitivity by blocking immune complex formation [2].

This unique class of immunoglobulins $\mathrm{G}$ has been shown to play a role in inducing immune tolerance in chronic

Correspondence: Marta Legatowicz-Koprowska, MD, Department of Pathology, National Institute of Geriatrics, Rheumatology and Rehabilitation, 1 Spartanska St., 02-637 Warsaw, Poland, e-mail: mlkoprowska@ gmail.com Submitted: 14.10.2016; Accepted: 19.01.2017 
or recurrent antigen exposure, such as in beekeepers or in patients receiving allergen desensitization therapy [3]. The proportion of IgG4 among all serum immunoglobulins $\mathrm{G}$ in these individuals increases markedly, i.e. up to $80 \%$, while normally, this proportion is $2-3 \%$. Unlike in other subclasses of $\mathrm{IgG}$, serum IgG4 levels vary significantly in healthy individuals, ranging from approximately $1 \mathrm{mg} / \mathrm{dl}$ to $140 \mathrm{mg} / \mathrm{dl}$ (35-51 mg/dl on average), nonetheless, few healthy individuals have IgG4 levels above $200 \mathrm{mg} / \mathrm{dl}$. Generally, higher serum IgG4 levels are found in males and the elderly [1].

The anti-inflammatory or immune tolerance-inducing mechanisms described above suggest a regulatory function of IgG4, but also show that specific exogenous antigens may induce a response by IgG4-positive B-cells. The role of endo- and exogenous specific antigens in the etiopathogenesis of IgG4-RD is currently a subject of studies and discussion. The response of IgG4-positive B-cells has been shown to be polyclonal, i.e. directed against multiple antigens [3]. The role of IgG4 more as an active witness than a cause of inflammation has been also suggested by the presence of multiple IgG4-positive cells in inflammatory infiltrations in nonspecific inflammatory conditions, inflammation surrounding neoplasms, or in some patients with autoimmune conditions (e.g. rheumatoid arthritis, systemic lupus erythematosus) [4].

It is worth noting the dissimilarities in pathogenetic mechanisms of autoimmune diseases and IgG4-RD. In the former, immunization processes and the production of the relevant inflammatory cytokines: interleukin 2 (IL-2), interferon $\gamma$, TNF- $\alpha$, are mediated by T helper 1 (Th1) or T helper 17 (Th17) cells. In contrast, IgG4-RD is due mainly to a response by $\mathrm{T}$ helper 2 (Th2) cells (cytokines: IL-4, IL-5, IL-13, and IL-21) and regulatory T-cells (Treg) (IL-10, TGF $\beta$ ). IL-10, known as an anti-inflammatory cytokine, causes B-cell differentiation into plasma cells and subsequent IgG4 production. IL-4 induces macrophages to produce high levels of IL-10. In turn IL-21, produced by Th2 and follicular T helper cells, is involved in the formation of germinal centers within lymphoid nodules and stimulates $\mathrm{IgG} 4$ production and infiltration of the affected tissues by IgG4-positive cells. Inflammatory monocytes also play an important role in ectopic lymphoid follicle formation in IgG4-RD. The affected organs enlarge as a result of massive infiltration by inflammatory cells and, often, the formation of secondary lymphoid follicles. Increased immunoglobulin production by numerous plasma cells leads to hypergammaglobulinemia.

Th2-cells also induce an allergic response with eosinophilia and elevated serum IgE levels. Activated, strongly IgE-positive mast cells promote naïve T-cell differentiation into Treg; as a result, they potentiate the effect of IL-10 [5]. This is a likely reason why $30-50 \%$ of patients with IgG4-RD have a history of concomitant or past allergies, eosinophilia, and increased IgE levels.

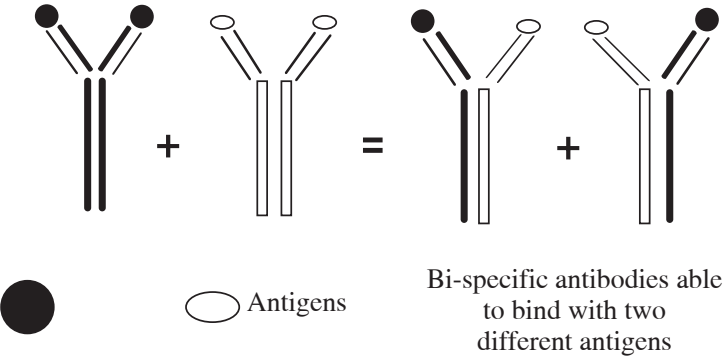

Fig. 1. Exchange of one of the two Fab fragments between IgG4 molecules

Recent studies indicate that the number of circulating $\mathrm{CD} 38^{\text {high }} \mathrm{CD} 27^{\text {high }} \mathrm{CD} 19^{+} \mathrm{CD} 2{ }^{-} \mathrm{CD} 22^{-}$plasma cells (shortlived immunoblasts) is elevated in patients with IgG4-RD, including those with normal serum IgG4 levels. Assessing the number of plasmablasts may be a better marker in disease diagnostics and disease course monitoring, including recurrence prediction [6].

The pathogenesis of fibrosis, responsible for organ enlargement and dysfunction may involve multiple factors. These include Treg-derived TGF $\beta$-1, which activates fibroblasts and induces endothelial and epithelial cell differentiation into myofibroblasts, resulting in tissue fibrosis [7].

There is more and more evidence suggesting underlying genetic mechanisms of IgG4-RD. Already in 1967, there was a case report of closely related males who simultaneously developed: retroperitoneal fibrosis, mediastinal fibrosis, sclerosing cholangitis, Riedel's thyroiditis, and orbital pseudotumors [8]. Studies of the last decades have indicated an association between the DRB1*0405 and DQB1*0401 HLA serotypes and AIP1. Patients with certain forms of IgG4-RD exhibit a gene polymorphism for: cytotoxic T-cell antigen 4 (CTLA4), TNF- $\alpha$, and Fc receptor-like 3 (FCRL3) genes [3].

\section{Epidemiology}

As a recently defined condition, IgG4-RD has yet to be subject to reliable epidemiological studies. However, males are already known to be affected more commonly than females. It is only in the case of head and neck involvement that the proportion of the affected individuals is nearly the same in both sexes. The mean age of symptomatic onset is the sixth decade of life, although the disease may affect younger individuals, and even children [9].

\section{Diagnosis}

Depending on the organ/system affected, IgG4-RD may have highly varied clinical manifestations. Elderly males constitute a majority of patients. Symptoms intensify slowly, over months, and even years. The course of the disease may have periods of spontaneous improvement or the symptoms may steadily persist on the same level. In such 
patients, diagnosis can be made after another/subsequent organ becomes involved.

Symptoms of IgG4-RD include diffuse pain of joints, tendons, with associated fatigue, especially when several systems/organs are already affected. There is no fever, night sweats, or - generally - weight loss.

In most cases, tissue biopsy is the gold diagnostic standard. Here, the role of fine-needle biopsy is greatly outweighed by biopsy involving a fragment of tissue (not only a group of cells), as a tissue fragment may reveal not only the presence of numerous IgG4-positive cells, but also morphological features, such as the characteristic, storiform pattern of fibrosis, as well as evidence of obliterative phlebitis. Individual organs have different established diagnostic numbers of IgG4-positive cells visible per high power field [10]. It is also important to assess the proportion of IgG4-positive cells among all IgG-positive cells, which should be at least $40 \%$ in most organs. Inflammatory infiltrates may contain eosinophils. Secondary lymphoid follicle formation is also observed. Definitive diagnosis may require analyzing several tissue fragments or even repeated biopsies. Histopathological differentiation is hindered by the already mentioned presence of numerous IgG4-positive cells in tissues in some nonspecific inflammatory conditions, autoimmune diseases, or around neoplasms.
Isolated high serum IgG4 levels are neither sufficiently sensitive nor sufficiently specific to establish the diagnosis, although IgG4 may be a helpful screening marker. Approximately $20 \%$ of patients with IgG4-RD have normal serum IgG4 levels, on the other hand, the levels may be also elevated in healthy individuals, even up to $200 \mathrm{mg} / \mathrm{dl}$. Serum IgG4 levels exceeding $135 \mathrm{mg} / \mathrm{dl}$ are generally considered to be diagnostically significant.

Nevertheless, histopathological evidence, even in combination with elevated serum IgG4 levels, does not determine a definitive diagnosis of IgG4-RD.

It is always necessary to consider the clinical presentation together with findings of imaging studies (ultrasound, MRI, PET). For example, the clearly consistent clinical and radiographic evidence may be sufficient for a diagnosis of IgG4-related AIP1. In the case of other organs, imaging study findings are not specific and require histopathological confirmation. PET is particularly useful in showing the involvement of other (asymptomatic in a given period) organs; it may be also useful in treatment monitoring.

Table 1 presents practical diagnostic guidelines for non-specialists regarding IgG4-RD diagnosis [9-11].

Neoplastic disease must be excluded in the differential diagnosis. Differential diagnosis for individual organs has been presented below.

Table 1. Practical diagnostic guidelines of IgG4-RD, based on Vasaitis - modified [9-11]

\section{Suspicion of IgG4-RD}

Clinical and/or radiological findings (swelling of organ or soft tissue masses), history of ongoing atopic disease

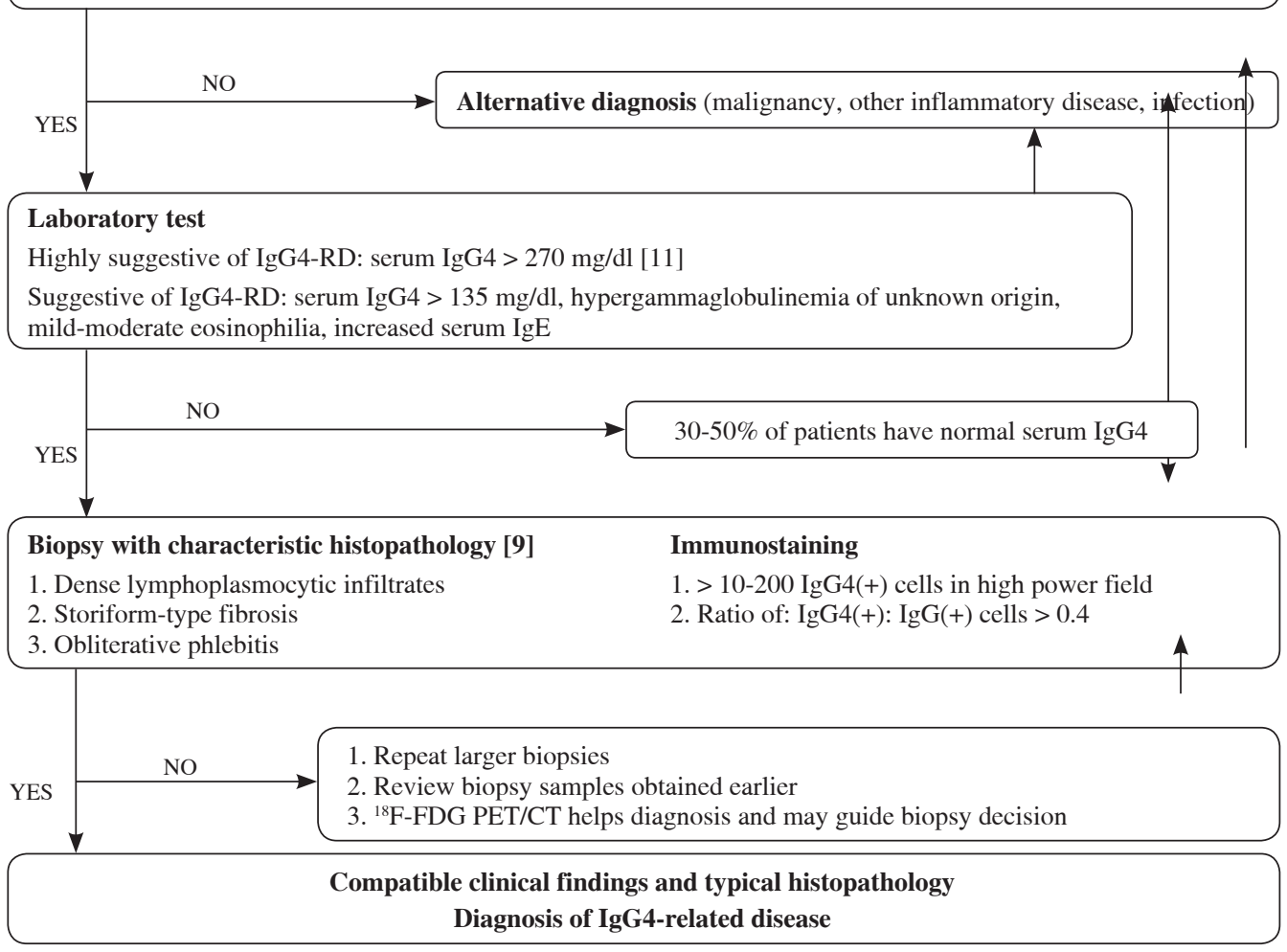




\section{Individual organ involvement}

\section{Pancreas and the biliary tract}

High levels of serum IgG4 were first detected in AIP1. The most common manifestation of this disease is obstructive jaundice (without any associated pain or fever) as a result of pancreatic edema due to inflammation and the resulting compression of the common bile duct or caused by concomitant IgG4-related sclerosing cholangitis.

Approximately half of the patients develop secondary diabetes mellitus, which makes the glucocorticoid (GC) therapy, recommended for IgG4-RD, problematic.

The most important aspect of differential diagnosis is to exclude pancreatic or bile duct cancer. Imaging studies, including endoscopy, can be helpful; however, they do not always provide definitive answers. Prior to empirical GC administration, it is recommended to perform an ultrasound-guided fine-needle pancreatic biopsy or a transpapillary biopsy during bile duct and pancreatic duct endoscopy, although excluding neoplastic growth may be impossible even via these methods (potential for sample error). There have been several reports of cases of pancreatic cancer in patients with AIP1.

\section{Salivary and lacrimal glands}

Chronic or recurrent bilateral parotid and/or submandibular gland enlargement and lacrimal gland enlargement with swollen upper eyelids, which change the appearance of the face, as reported by J. Mikulicz-Radecki in 1888, are characteristic for IgG4-RD. This form of the disease affects mostly men, typically with submandibular gland involvement, without the burdensome sicca syndrome or the presence of anti-SS-A and anti-SS-B antibodies, which differentiates this condition clinically from Sjögren's syndrome. The diagnosis is confirmed by detecting elevated serum IgG4 levels and histopathological findings consistent with chronic sialadenitis with rich infiltration of IgG4-positive plasma cells, not meeting the focus score criterion or exhibiting adenoid tissue damage observed in Sjögren's syndrome.

\section{Retroperitoneal fibrosis}

For decades known as Ormond's disease and currently classified as a form of IgG4-RD, idiopathic retroperitoneal fibrosis may manifest as peri-aortitis (including aortic wall involvement with dissecting aneurysm formation), peri-ureteral fibrosis (with potential ureteral obstruction and hydronephrosis), or the presence of abnormal extensive tissue masses in the retroperitoneal space. The clinical manifestations, including difficult to pinpoint pain in the back, flanks, hypogastrium, or thighs and lower limb edema, rarely suggest the diagnosis directly. Histological findings from tissues collected typically already at a late stage of the disease more commonly show prevalent fibro- sis and/or obliterative phlebitis, rather than inflammatory infiltrations with a large proportion of IgG4-positive cells, as the latter may have already resolved. Inflammation and fibrosis may also affect the thoracic aorta, or smaller-caliber vessels - e.g. coronary arteries [9].

\section{Lymph nodes}

Local or systemic lymph node involvement in the course of IgG4-RD manifests as their painless enlargement and always requires differentiating from lymphoma, metastases, infection, and Castleman disease. Histopathological confirmation of the diagnosis is difficult because the fibrosis pattern characteristic of IgG4-RD is not typically found in lymph nodes.

\section{Thyroid gland}

The first manifestation of IgG4-related thyroiditis may be hypothyroidism, and although thyroid enlargement may not be palpable, it is visible in imaging studies. Later, the thyroid becomes enlarged and fibrous, which may even lead to compression-induced dysphagia and dyspnea. Riedel's thyroiditis and Hashimoto's thyroiditis are also part of the IgG4-RD spectrum [12].

\section{Other locations}

There have been reports of various forms of the disease involving the following locations: the orbit and/or nose with paranasal sinuses, kidneys (interstitial nephritis), meninges (meningitis), the pituitary, lungs and mediastinum, prostate, skin, and peripheral nerves. Individual organ involvement requires differentiation from vasculitis (including granulomatosis, i.e. granulomatosis with polyangiitis), sarcoidosis, moreover - in nearly all locations - the disease must be differentiated from neoplastic growths (including lymphomas).

\section{Treatment}

Most IgG4-RD manifestations respond to therapy with GCs, which are a first-line treatment. It is recommended to initiate treatment with prednisolone at a daily dose of 0.6-1.0 mg/kg [13] and down-titrate the dose after approximately 2-4 weeks to a complete discontinuation or reach a low maintenance dose after 2-3 months.

The diagnosis is confirmed by a dramatic improvement if definitive histological examination was not earlier possible. A lack of response to GCs suggests that another diagnosis especially that of neoplastic disease should be considered. Effective treatment also leads to improved (pancreatic, salivary, etc.) gland function (unlike in Sjögren's syndrome). Unfortunately, much worse effects can be expected when tissues (the retroperitoneal space, mediastinum) already show significant fibrosis, which emphasizes the importance of early diagnosis. 
In order to avoid the unfavorable effects of long-term corticoid therapy, azathioprine, mycophenolate mofetil, or methotrexate can be used in the treatment. If no response to these agents is observed, rituximab, which targets B-cells, is administered. However, there are no clinical studies evaluating these treatments.

Certain clinical conditions may require emergency interventions, such as balloon dilatation or stenting of bile ducts or ureters, a vascular bypass, or aortic angioplasty. An orbital pseudotumor may require radiotherapy to achieve remission.

\section{Conclusions}

IgG4-RD is a newly classified and more and more commonly diagnosed condition involving inflammatory and fibrotic processes, and characterized by histological findings in various affected organs. A consensus has been reached regarding morphological diagnostic criteria [10]. However, there is a continuing debate as to the diagnostically significant value of serum IgG4 levels. It is still not uncommon for differential misdiagnoses to occur, these involve malignant neoplasms, lymphomas, Sjögren's syndrome, and other conditions depending on the location. Meanwhile, an accurate early diagnosis determines initiation of the effective GCs treatment, which helps prevent extensive fibrosis, with the resulting organ damage and, often dramatic, functional impairment.

The pathogenesis of IgG4-RD remains unclear. Further immunopathological studies are needed to explain the interactions between activation pathways of T-cells with various (including new) phenotypes, which induce cytokine-mediated IgG4 production. The role of IgG4 in the inflammatory and fibrotic process is still a subject of debate. The natural properties of IgG4 suggest its ability to regulate, and even inhibit immune reactions (both inflammatory and allergic). Further studies may help show whether IgG4 is the main culprit or only an active witness of the developing, often dangerous, changes in tissues.

\section{The author declares no conflict of interest.}

\section{References}

1. Nirula A, Glaser SM, Kalled SL, et al. (2011): What is IgG4? A review of the biology of a unique immunoglobulin subtype. Curr Opin Rheumatol 23: 119-124.

2. Umehara H, Okazaki K, Masaki Y, et al. (2012): A novel clinical entity, IgG4-related disease (IgG4RD): general concept and details. Mod Rheumatol 22: 1-14.

3. Smit W, Barnes E (2014): The emerging mysteries of IgG4-related disease. Clin Med 14 (Suppl 6): 56-60.

4. Strehl JD, Hartmann A, Agaimy A (2011): Numerous IgG4positive plasma cells are ubiquitous in diverse localised non-specific chronic inflammatory conditions and need to be distinguished from IgG4-related systemic disorders. J Clin Pathol 64: 237-243.
5. Takeuchi M, Sato Y, Ohno K, et al. (2014): T helper 2 and regulatory T-cell cytokine production by mast cells: a key factor in the pathogenesis of IgG4-related disease. Mod Pathol 27: 1126-1136.

6. Wallace ZS, Mattoo H, Carruthers M, et al. (2015): Plasmablasts as a biomarker for IgG4-related disease, independent of serum IgG4 concentrations. Ann Rheum Dis 74: 190-195.

7. Rosenbloom J, Mendoza FA, Jimenez SA (2013): Strategies for anti-fibrotic therapies. Biochim Biophys Acta 1832: 10881103.

8. Comings DE, Skubi KB, Van Eyes J, et al. (1967): Familial multifocal fibrosclerosis. Findings suggesting that retroperitoneal fibrosis, mediastinal fibrosis, sclerosing cholangitis, Riedel's thyroiditis, and pseudotumor of the orbit may be different manifestations of a single disease. Ann Intern Med 66: 884-892.

9. Vasaitis L (2016): IgG4-related disease: A relatively new concept for clinicians. Eur J Intern Med 27: 1-9.

10. Deshpande V, Zen Y, Chan JKC, et al. (2012): Consensus statement on the pathology of IgG4-related disease. Mod Pathol 25: 1181-1192.

11. Inoue D, Yoshida K, Yoneda N, et al. (2015): IgG4-related disease: dataset of 235 consecutive patients. Medicine 94: 1-8.

12. Jokisch F, Kleinlein I, Haller B, et al. (2016): A small subgroup of Hashimoto's thyroiditis is associated with IgG4-related disease. Virchows Arch 468: 321-327.

13. Kamisawa T, Zen Y, Pillai S, Stone JH (2015): IgG4-related disease. Lancet 385: 1460-1471. 